Chapter 2

\title{
Biopolymer-mediated Green Synthesis of Noble Metal Nanostructures
}

\author{
Olayemi J. Fakayode, Adewale O. Oladipo, Oluwatobi S. Oluwafemi and \\ Sandile P. Songca
}

Additional information is available at the end of the chapter

http://dx.doi.org/10.5772/62127

\begin{abstract}
Polymer-coated noble metal nanoparticles are currently of particular interest to investigators in the fields of nanobiomedicine and fundamental biomaterials. These materials not only exhibit imaging properties in response to stimuli but also efficiently deliver various drugs and therapeutic genes. Even though a large number of polymer-coated noble metal nanoparticles have been fabricated over the past decade, most of these materials still present some challenges emanating from their synthesis. The metal nanoparticles when encapsulated in a polymer and taken up by human cells might show a lower degree of toxicity; however, the degree of toxicity for some of the starting materials and precursors has raised serious concerns. Hence, there is a need to implement the principle of green chemistry in the synthesis of nanomaterials. The use of environmentally benign materials for the synthesis of metal nanoparticles provides numerous benefits ranging from biocompatibility, availability, cost-effectiveness, amenable scale-up to eco-friendliness. The biopolymer-based nanovehicles have been found to be more suitable in the field of nanotechnology owing to their high reproducibility, ease of manufacture, functional modification and safety (they are not carcinogenic). Unlike synthetic polymers where the raw material can be derived from petrochemicals or chemical industrial processes, biopolymers are produced from renewable resources such as plant and/or living organism. They are degradable by natural processes down to elemental entities that can be resorbed in the environment. Furthermore, they can also be modified to serve a particular purpose which explains the myriad of their potential applications. The macromolecular chain of these biopolymers possesses a large number of hydroxyl groups which can easily complex with metal ions. Additionally, these biopolymers also contain supramolecular structures that can lead to new functionalities of their composites with metal and semiconductor nanoparticles. In this chapter, a comprehensive discussion on different biopolymers, green synthesis of noble metal nanostructures, mechanisms, characterization and application in various fields is presented.
\end{abstract}

Keywords: Biopolymers, Nanoparticles, Green synthesis, Bionanocomposites, Noble metals 


\section{Introduction}

In our modern day, nanotechnology has continued to play a vital role in a plethora of biomedical and biotechnological applications especially in sensing, imaging and treatment of various diseases. A variety of noble metal nanostructures encapsulated or coated with biopolymers have been studied by researchers in the field of nanobiomedicine. This is due to the interesting properties and wide-spectrum application of biopolymer-based nanomaterials. These materials have been shown to combine both the intrinsic features of noble metal nanostructures and the biological features presented by renewable source polymers [1-3]. They have been found not only to exhibit excellent imaging properties but also to efficiently deliver various drugs and therapeutic genes [4-7]. Noble metal nanostructures used in therapy and diagnosis must be non-toxic, stable in biological media and should be specific for the target $[8,9]$. However, the requirement of these three factors has hindered the use of many noble metal nanostructures in a variety of biomedical applications. Hence, there is a need for the conjugation of these materials with functional biological molecules to necessitate and improve their efficacy for biomedical functionalities. Bionanocomposites exhibit better biomedical values than their naked nanoparticle counterparts. They demonstrate colloidal stability, maintain plasmonic properties and show little or no effect on cell viability in the biological cell system $[10,11]$.

Generally, the synthesis of most noble metal nanostructures requires a high concentration of surfactant, which directs the asymmetric geometry. However, studies have shown that surfactants tend to degrade biological membranes, thus raising significant concern about the cytotoxicity of these materials [12,13]. The cytotoxicity of these surfactant-bound nanostructures can be reduced by minimizing the surfactant concentration below the critical micellar concentration. This reduction is effected at the expense of the stability of the nanomaterials solution, and as a result, their unique optical properties in biological environments are compromised.

There have been calls for the development of nanomaterials based on the principles of green chemistry and, consequently, a myriad of studies have emerged. Some of the proposed solutions are based on the substitution of toxic reagents with more eco-friendly materials. As a result, a lot of research has been done on developing more environmentally benign synthetic methods for noble metal nanomaterials owing to their wide area of applications. In addition, there have been calls for the development of sustainable process and practices in order to define products as green based.

Raveendran and co-workers [14] report three main steps to be considered in the green synthesis of metallic nanoparticles. These are (i) the choice of solvent medium used for the synthesis, (ii) use of environmentally benign reducing agent and (iii) the choice of non-toxic material as stabilizing agent. The use of environmentally benign materials for the synthesis of metal nanoparticles provides numerous benefits ranging from biocompatibility, renewable feedstocks, cost-effectiveness, waste prevention, amenable scale-up, synthetic steps reduction, use of safer solvents and increase of energy efficiency to eco-friendliness [15-17]. 


\subsection{Significance and limitations of nanomaterials}

Nanomaterials possess phenomenal ability to create better materials as they are currently being used in numerous products and industrial applications (Figure 1). Most of these materials present evolutionary development of existing technologies. The use of biomaterials such as ascorbic acid, glutathione, sugars, glycerol, orange peel, plant extracts and yeast, as alternative raw materials for synthesizing nanomaterials presents an even more fascinating approach, thus impacting their significance [18,19]. In the area of nanomaterial research, noble metal nanostructures have received tremendous attention due to their wide range of applications in electronics, sensing, catalysis, photonics, environmental clean-up, imaging, water purification, cancer therapy, labelling and drug delivery [20-23]. Most notable among the breakthrough applications of biopolymer-based noble metal nanostructures include: the use of biopolymerbased biodegradable food-packaging materials in the place of non-biodegradable plastics [24], nanocrystal metal-chitosan granular composite material for water purification [25], selective tumour ablation using polymer-coated gold nanorods and metal nanostructure for sensing applications [26-28].

Nevertheless, with noble metal nanostructures having such a phenomenal advantage, there also exist limitations to their application. There have been increasing concerns relating to their toxicity arising from their bioaccumulation in human body. It has been reported that they preferentially accumulate in the liver and spleen [29,30]. As a result, they cause many dysfunctions to these organs causing disruption to their body activities. The nanoparticles size, shape and surface functional groups play a vital role in their toxicity effect. There exist a challenge of conjugating nanoparticles to the surface of metal nanoparticles with negligible or no toxicity effect. As the nanoparticles become conjugated, the surface chemistry, size and shape tend to change, thus influencing their cell and protein interface interactions. This inevitably leads to unspecific and non-selective activities in the body [31].

\subsection{Noble metal nanoparticles}

Nanoparticles of gold, silver and platinum are classified as noble metal nanostructures. They are nanosized metals with at least one dimension within the nanometre size range of 1-100 $\mathrm{nm}$. Unlike bulk metals, which are typically ductile and possess high thermal and electrical conductivity, metal nanoparticles completely differ in such physical properties. The properties exhibited by metal nanoparticles are completely different from those exhibited by their corresponding bulk metals due to the absence of electron delocalization [32]. Noble metal nanoparticles have very large surface-area-to-volume ratio when compared to their bulk counterparts, thus making them attractive for a wide array of many applications. Their enhanced properties can be tailored by controlling their shape, size and composition [33,34]. A new generation of hybrid nanostructured materials presents an emerging field in nanotechnology. Nanobiocomposite over the past few years has become a term used to designate composites, which contain naturally occurring polymers (biopolymers) and an inorganic structure. The development of nanocomposites comprising a biopolymer matrix with noble metal nanostructures has been extensively studied and considerable efforts are now being directed at biopolymer-based nanocomposites with improved properties $[25,26]$. 


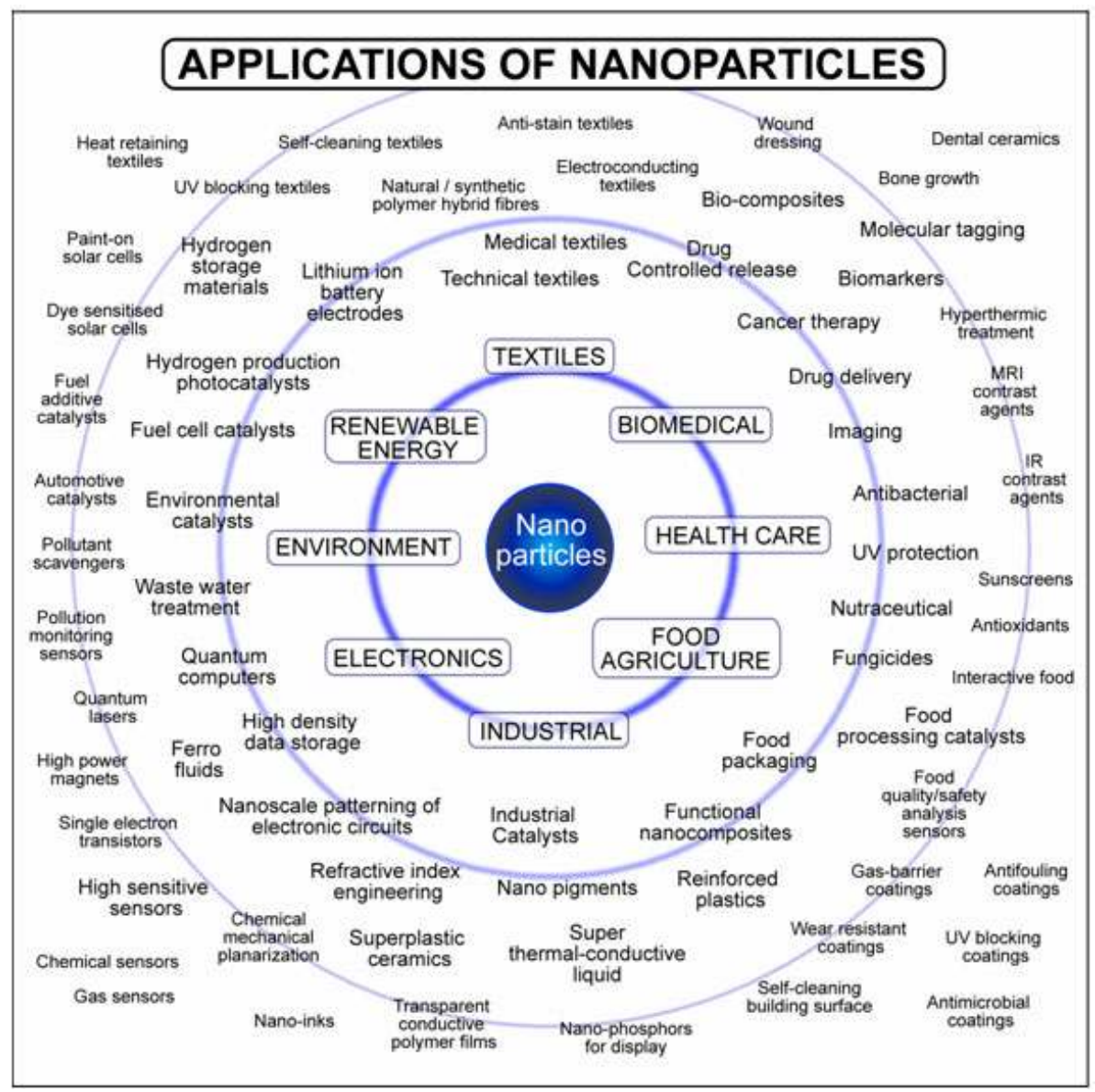

Figure 1. Applications of nanoparticles [134].

\section{Synthesis and characterization of noble metal nanoparticles}

Nanoparticle synthesis over the past decades has received enormous attention due to their extensive applications. Today, researchers have been able to controllably synthesize noble metal nanoparticles of various shapes and dimensions. These include zero-dimensional, onedimensional, two-dimensional and three-dimensional nanostructures in a remarkable fashion [35-40]. Noble metal nanoparticles have been synthesized using various methods. Some of these methods include the electrochemical method [41], photochemical method [42], chemical reduction method [33], sonochemical method [43] and the biosynthesis method [44]. A universal reflection on the environmental risks or hazards and sustainability arising from most of these methods other than biosynthetic routes prompted the need for greener approaches in the manufacture of these materials. As a result, considerable attention has been focused on the 
biosynthesis as the method of choice for the synthesis of these nanoparticles. Metal nanoparticles have been embedded in a host biopolymer matrix in a variety of ways, which include in situ chemical synthesis, plasma polymerization combined with metal evaporation, ion implantation and vacuum deposition on viscous flow polymer.

\subsection{Synthesis using non-polysaccharide reductants}

Interesting biopolymers have been used to synthesize gold, silver and platinum nanoparticles via direct in situ reduction [45]. This method enables rapid nucleation and growth of noble metal nanoparticles embedded in a biopolymer matrix using non-polysaccharide reductants. The result is either nanoparticle composites or more complex nanostructures, depending on the reaction conditions used. The non-polysaccharide reductant can be either strong like sodium borohydride or mild like sodium citrate and tetrakis(hydroxymethyl)phosphonium chloride (THPC).

\subsubsection{Sodium citrate}

The use of sodium citrate in the synthesis of gold and silver nanoparticles is perhaps the first method to be developed [46] and it is still relevant today. It is a simple process for reducing metal salts to nanoparticles with modest monodispersed spherical shape. The particle diameters are usually within the range of 10-20 nm. Colloidal gold and silver nanoparticles are produced by this technique because the citrate ions act as both capping and reducing agents. Following this trend, spherical silver nanoparticles with monodispersed and controlled sizes have also been effectively synthesized. In addition, monodispersed gold nanoparticles with controlled size have also been reported. This is achieved by altering the concentration of gold chloride ions with respect to the citrate [47]. Furthermore, Bastus and co-workers [48] report the enhanced citrate-reducing ability in combination with tannic acid. The tannic acid is instrumental to the formation of the seeds and subsequent high yield of the final spherical particles.

\subsubsection{Sodium Borohydride $\left(\mathrm{NaBH}_{4}\right)$}

The most common chemical reduction method for preparing noble metal nanoparticles involves the reduction of their metal salts or precursor (from $\mathrm{M}^{+\mathrm{n}}$ to $\mathrm{M}^{0}$ ) using sodium borohydride as the reducing agent. Sodium borohydride is a strong reducing agent in organic and inorganic chemistry, and it is used to reduce most transition metal ions to zero-oxidation metal nanoparticle state $\mathrm{M}(0)$ in the presence of a colloidal stabilizer [49]. The stabilizing agent prevents the aggregation of the nanoparticles after formation. $\mathrm{NaBH}_{4}$ reduces metal precursors by hydride transfer. However, single-electron transfer is also a possibility, given the electronrich nature of borohydride anion. Sodium borohydride can also act as bi- or tridentate ligands [50]. It has also been demonstrated that $\mathrm{NaBH}_{4}$ has the ability to control the size and morphology of nanoparticles to a large extent using appropriate synthetic techniques. As a result, nanoparticles with very large surface area relative to volume have become feasible. This is particularly important because the surface plasmon resonance (SPR) band of nanoparticles is a function of morphology [51,52]. The concentration of $\mathrm{NaBH}_{4}$ has been found to affect the 
size, shape, dispersity and even hydrodynamic diameter of the metal nanoparticles. As the concentration of borohydride increases, the aspect ratio (length divided by width) of the nanorods formed decreases. Moreover, at a certain higher concentration, shape deformation occurs. Also, ageing the sodium borohydride solution prior to use has been found to influence the shape formation of the nanorods [53]. Monodispersed Ag-NPs have also been synthesized using borohydride reduction of silver salt. The borohydride anions are adsorbed onto silver nanoparticles and aggregation is prevented by polyvinyl pyrrolidone (PVP) addition. A large excess of the reductant is also needed to stabilize the nanoparticle product [49] since they are adsorbed onto silver nanoparticles after they are formed.

\subsubsection{Tetrakis (Hydroxymethyl) Phosphonium Chloride (THPC)}

THPC is an exceptionally strong reducing agent capable of synthesizing smaller gold or silver nanoparticles usually used as seeds for the formation of nanoshells [54] and gold nanoclusters employed for fluorescence imaging [55,56]. Typically, it serves as a good reducing agent in alkaline solution. However, to achieve good nanoparticle stability, a stabilizer is often added alongside with THPC during synthesis. Nonetheless, Hueso and co-workers have reported the use of THPC as both reducing agent and stabilizing ligand in the synthesis of ultra-small noble metal nanoparticles. This is achieved in a single-step room-temperature method with a wide variety of monometallic and bi/tri nano alloys as products [57]

\subsection{Synthesis using polysaccharide reductants}

Polysaccharides are long polymers of monosaccharide sugars and their derivatives. Unlike proteins or nucleic acids, these polymers can either be straight chained or branched. They can be of one type of monosaccharide (homopolysaccharides), or more than one (heteropolysaccharides) in repeating units. Polysaccharides can also be divided into groups according to their two major functions: contribution to structural components of cells and energy storage. Cellulose is a structural polysaccharide while starch is mainly an energy storage polysaccharide. Starch is the main energy storage in plants while glycogen is the main energy storage in animals. Both starch and cellulose are polymers of glucose but they differ based on the configuration of $\mathrm{C}-\mathrm{O}$ bonding [58]. They are generally insoluble in cold water, although at around $100{ }^{\circ} \mathrm{C}$, starch is water-soluble while cellulose needs a temperature higher than $300{ }^{\circ} \mathrm{C}$ to be water-soluble [59]. Upon hydrolysis with acids or enzymes, they eventually yield their constituent monosaccharide sugars. Polysaccharides have the ability to coordinate metal ions and thus act as stabilizing agents. Furthermore, they have been known to exhibit reducing properties in the synthesis of metal nanostructures. This dual role has enabled the production of metal nanoparticles with improved properties and functionalities such as different shapes and sizes, hydrophilicity, biocompatibility, specificity and non-toxicity. Due to their non-toxic nature, polysaccharides are recognized as green reducing agents for nanostructure synthesis. Their structural hydroxyl groups provide them with strong reducing ability and solubility in water. Other natural polysaccharides such as chitosan have also gained attention in the preparation of nanoparticles [60]. Under this section, we will review the use of starch, cellulose and dextran for the synthesis of noble metal nanoparticles. 


\subsubsection{Starch}

Chemically, starch consists of long chains of D-glucose (a monosaccharide) units joined by 1,4glycosidic bonds. They are formed in plants during photosynthesis. They are present in many plant-based food sources such as root vegetables, for example, potatoes and cereals. The ecofriendly biosynthesis of metal nanoparticles using starch has been well reported. Most of them have been performed through reduction processes. It is a simple method which yields high amount of nanoparticles and to some extent allows for control of particle size. El-Rafie and coworkers [61] report the use of alkali-treated maize starch as both reducing agent and stabilizer for the production of silver nanoparticles. In their report, a redox reaction occurs between $\mathrm{AgNO}_{3}$ and alkali-treated starch to obtain the nanoparticles. The nanosilver obtained is spherical in shape with particle size ranging between 4 and $6 \mathrm{~nm}$. Valencia and co-workers [62] also report the use of starch as reducing agent at $90^{\circ} \mathrm{C}$ in the presence of $\mathrm{NaOH}$. Sphericalshaped Ag-NPs with particle diameter between 10 and $30 \mathrm{~nm}$ were produced. Furthermore, the role of $\mathrm{pH}$ in the green-mediated synthesis of starch-capped Ag-NPs has been investigated. Ag-NPs are synthesized using two green materials: glucose as the reducing agent and starch as the stabilizing agent. The presence of an accelerator $(\mathrm{NaOH})$ is found to affect the size, size distribution and the $\mathrm{pH}$ of the solution of the as-synthesized Ag-NPs. At mildly acidic conditions, occurrence of starch hydrolysis affects the particle size. The insufficient protection of the particle surface led to the formation of larger particles but with defined sizes. However, less starch hydrolysis results in nanoparticle aggregation leading to a blue shift and splitting of the spectra [63]. Spherical nanoparticles and nanowires of gold and silver with various sizes and shapes have also been prepared using a monosaccharide (D-glucose) and a polysaccharide (soluble starch) [64].

Gold nanoparticles coated with different sugars are successfully synthesized using a non-toxic, water-soluble phosphine amino acid (THPAL) as a reducing agent. The sugars used are glucose (monosaccharide); sucrose, maltose or lactose (disaccharides); raffinose (trisaccharide) and starch (polysaccharide) [65]. The capping ligand plays a vital role in transforming the shapes of the nanoparticles formed. Platinum nanoparticles (Pt-NPs) with uniform particle size (2-4 $\mathrm{nm}$ ) have also been synthesized using soluble starch. The soluble starch performs a dual role as both reducing and stabilizing agents. Under alkaline treatment, the degraded intermediate species with reducing potentials (i.e., small molecules of aldehydes and hydroxyl ketones) generated in situ, completely reduces the platinum ions. The hydroxyl group in the starch structure complexes with the platinum ions and prevents aggregation or precipitation, thus sufficiently stabilizing the platinum nanoparticles formed [66]. Furthermore, the use of glucose as a reducing agent and starch as stabilizing agent to protect Pt-NPs core in buffer solution have also been reported [67].

\subsubsection{Cellulose}

Cellulose chemically consists of long chains of six-membered ring glucose (a monosaccharide) molecules. The repeating units comprise two anhydroglucose rings $\left(\mathrm{C}_{6} \mathrm{H}_{10} \mathrm{O}_{5}\right) n$, where $n=10,000-15,000$. They comprise the fibre and are indigestible by humans but digestible by some other animals [68-70]. Celluloses are ideal materials upon which new biopolymer 
composites have thrived. For instance, carboxymethyl cellulose (CMC) obtained from cotton have been used in the synthesis of Ag-NPs at different conditions. The degree of polymerization of CMC is shown to have significant impact on the reduction capacity, particle size and stability of the silver nanoparticles formed [71]. Also, synthesis of Ag-NPs has been carried out with cellulose playing a dual role of both reducing and capping agent in aqueous medium. The cellulose is extracted from an environmentally problematic aquatic weed, water hyacinth. Particle shape and size evolutions of the silver nanoparticles are achieved by varying the $\mathrm{pH}$ of the solution and reaction time [72]. Metal nanoparticles of gold, silver and platinum have also been prepared using cellulose gel. Filter paper pulp is used to generate the cellulose solution which was allowed to coagulate to form a gel. The nanoparticles of gold, silver and platinum formed are well dispersed and stabilized by the cellulose network. The nanoporous structure of the cellulose hydrogels also prevents aggregation of the nanoparticles formed [73]. Some bacteria are also capable of producing cellulose via digestion. Cellulose of this nature is different from cellulose obtained from trees and cotton because it is free from lignin and hemicellulose. Colloidal silver nanoparticles could be prepared using cellulose of bacterial origin. Bacterial cellulose (BC) is immersed in the silver nitrate solution and reduced by different reducing agents (ascorbic acid, hydrazine and hydroxylamine) under the influence of gelatine and polyvinyl pyrrolidone (PVP), as colloidal stabilizers. The particle size and size distribution of the $\mathrm{BC}-\mathrm{Ag}^{0}$ composite depend on the reductant type and stabilizer used. Best results are obtained for ascorbic acid-reduced/gelatine-protected $\mathrm{Ag}$ composite [74].

\subsubsection{Dextran}

Dextran are water-soluble branched polysaccharides of glucose (dextrose) produced by lactic acid bacterial action on sucrose. The linear chains are linked by $\alpha-1,6$ glycosidic bond between glucose molecules while the branches begin from $\alpha-1,3$ linkages. In this method, metal nanostructures are prepared using an environmentally benign solvent and dextran as capping agent, or in some cases dextran serves as both reducing and capping agents. Wang and coworkers [75] report the synthesis of well-dispersed, uniform and biocompatible gold nanoparticles at room temperature using dextran as both reducing and stabilizing agent. The assynthesized dextran-coated Au-NPs are stable in high ionic strength medium, thereby making them important materials for fabricating novel nanostructures.

Environmentally friendly copolymers of dextran have also been used to synthesize goldcopolymer and silver-copolymer nanoparticles. The simple green synthetic method makes use of graft copolymers, dextran-graft-poly( $\varepsilon$-caprolactone) and dextran-graft-poly( $\delta$ valerolactone) as reductants and stabilizers in the synthesis of the noble metal nanoparticles. The amphiphilic nature of the copolymer is crucial during the process, as this allows interaction of the copolymer with the metal surface and the aqueous medium. The $\mathrm{OH}$ end groups of the grafted blocks of copolymers are responsible for the reduction of gold and silver ions [76,77]. 


\subsection{Characterization of polysaccharide-stabilized gold and silver nanoparticles}

\subsubsection{UV-Visible spectrophotometry}

UV-Visible spectrophotometry is an essential powerful tool for characterizing the optical properties of various materials at molecular and atomic levels. Typically, gold and silver nanoparticles exhibit a UV-Visible characteristic absorption band peak around 520 and 400 $\mathrm{nm}$, respectively, due to their SPR properties [78-81]. The SPR is due to the collective oscillation of light-excited free-moving conduction band electrons present at the surface of the noble metals. Stabilizing these metals with polysaccharides usually causes a change in the electron density of the metals and thus a shift in the positioning of their SPR peaks. However, the direction and the actual positioning of SPR peak depend on the type of nanoparticle $[79,80]$, particle size and structure of polysaccharide involved [82-84].

\subsubsection{Fourier Transform Infrared Spectroscopy (FTIR)}

FTIR is used to identify the functional groups present in the polysaccharide used to stabilize metal nanoparticles [80]. In conjunction with UV-Visible, dynamic light scattering and Zeta potential data, FTIR can be used to evaluate the extent of polysaccharide stabilization of metal nanoparticles (NPs). Stereotypically, polysaccharide-stabilized gold or silver NPs usually show absorption wavenumber bands for $\mathrm{O}-\mathrm{H}$ (3500-3200 $\mathrm{cm}^{-1}$ : alcohols; 3300-2500 $\mathrm{cm}^{-1}$ : acids), C-H (3000-2850 $\mathrm{cm}^{-1}$ : saturated alkanes) and C-O (1320-1000 $\mathrm{cm}^{-1}$ : alcohols and ethers) groups associated with general carbohydrate structure $[79,80,83,85]$. To ascertain the effectiveness of stabilization of nanoparticles by the polysaccharide, shifts in the affected groups are usually observed. The hydroxyl, hemiacetal and aldehyde groups play significant roles in the synthesis and stabilization of polysaccharide-capped silver $[85,86]$ and gold nanoparticles $[79,83]$.

\subsubsection{Zeta potential}

The zeta potential measures the overall charge on polymer-stabilized noble metal nanoparticle system [87] as either negative or positive in millivolts. As mentioned earlier, it may be used in conjunction with FTIR to assess the extent of nanoparticle stabilization by polysaccharides. When stabilized with carboxylic acid-terminated polysaccharides, gold and silver NPs are usually surrounded by negative charges. The negatively charged polysaccharide molecules interact with one another via electrostatic repulsion and thus help to prevent agglomeration. Similarly, positively charged polysaccharide-stabilized gold and silver nanoparticles are stabilized via electrostatic repulsion of the polysaccharide's molecules. The higher the magnitude of the zeta potentials, the greater is the extent of charge-to-charge electrostatic repulsion and thus the greater is the stability of nanoparticles [82] and vice versa. The negatively or positively charged polysaccharide-stabilized silver nanoparticles are important for efficient antimicrobial efficacy of silver nanoparticles against gram-positive and gramnegative microbes, respectively [82]. 


\subsubsection{Dynamic Light Scattering (DLS)}

The dynamic light scattering, also known as the photon correlation spectroscopy, uses light scattered by moving particles in a dispersible solution system to determine the hydrodynamic size distribution of various particles present in the solution. DLS particle-size estimation is based on measurement of the intensities of scattered lights by various particles present in the solution, provided the particles' movement is Brownian in nature. Therefore, it is used to evaluate the homogeneity of a solution and thus the aggregation of nanoparticle in the solution. For a reliable DLS measurement, a polydispersity index of between 0.05 and 0.7 may be preferable. Also, the refractive indices $(n)$ and absorption values $(k)$ of both the material and solvent must be accurately known for a reliable conversion of intensity data to volume or number size distribution. By default, particle sizes are obtained from the extrapolation from the intensity's data. However, data can be converted to obtain particle sizes relative to volume (size based on concentration of particles) or number of primary particles (size based on percentage number of primary particles) using Mie formula. The most important use of DLS's characterization of polysaccharide-stabilized gold and silver nanoparticles is to evaluate the extent of size characteristics after stabilization. For example, it has been reported that particle size decreases with increasing concentration of polysaccharide $[80,83]$ up to a certain extent and then increases at higher concentration due to aggregation [80]. Thus, DLS can be used to obtain the optimal concentration of the stabilizing agent that favours non-aggregation of particles in a given solution [83]. DLS can also be used to evaluate the effect of the swelling of polymers on the size of nanoparticles when the temperature of the medium is increased.

\subsubsection{Transmission Electron Microscopy (TEM)}

TEM is a two-dimensional technique used to probe the shape and morphology of a nanoparticle system $[83,88]$. The shapes of nanoparticles affect the optical, chemical and biological properties of metal nanoparticles. For example, silver nanoparticles have been shown to evolve different shapes as temperature increases when synthesized and stabilized by methyl cellulose [89]. This change in shape morphology of stabilized silver nanoparticles was observed using the TEM technique (Figure 2). Furthermore, TEM can be used to estimate the particle-size distribution of nanoparticle systems [86]. The particle-size data obtained from TEM are usually compared to the information obtained from other particle-size measurements such as DLS and X-ray diffractometry for analytical reliability and accuracy. Furthermore, TEM can be used in conjunction with FTIR, UV-Vis and other probing techniques to elucidate the mechanism of evolution of nanoparticles during the course of the synthesis (Figure 3).

\subsubsection{Scanning Electron Microscopy (SEM), Inductively coupled Plasma Spectroscopy (ICP-MS) and powder X-Ray Diffractometry (XRD)}

SEM is similar to TEM but differs in its magnifications and image outputs. SEM is a threedimensional imaging probe, which can be used to visualize the three-dimensional images of stabilized nanoparticles $[80,90,91]$ (Figure 2). ICP-MS is mainly used to determine the concentration of metals in a material. Thus, it can be used to estimate the concentration of gold and silver nanoparticles [92] within the surrounding structure of polysaccharide stabilization. The 
amount of nanoparticles present within a stabilizing matrix is essential for such sensitive applications such as environmental sensing, industrial catalysis and biomedical diagnostics and therapies. The powder XRD technique is used to probe the extent of the crystallinity of both unstabilized and stabilized nanoparticles. Particles size can also be estimated using XRD technique. Usually, a face-centred cubic structure is observed for polysaccharide-stabilized gold $[79,83,93]$ and silver nanoparticles $[82,90]$.
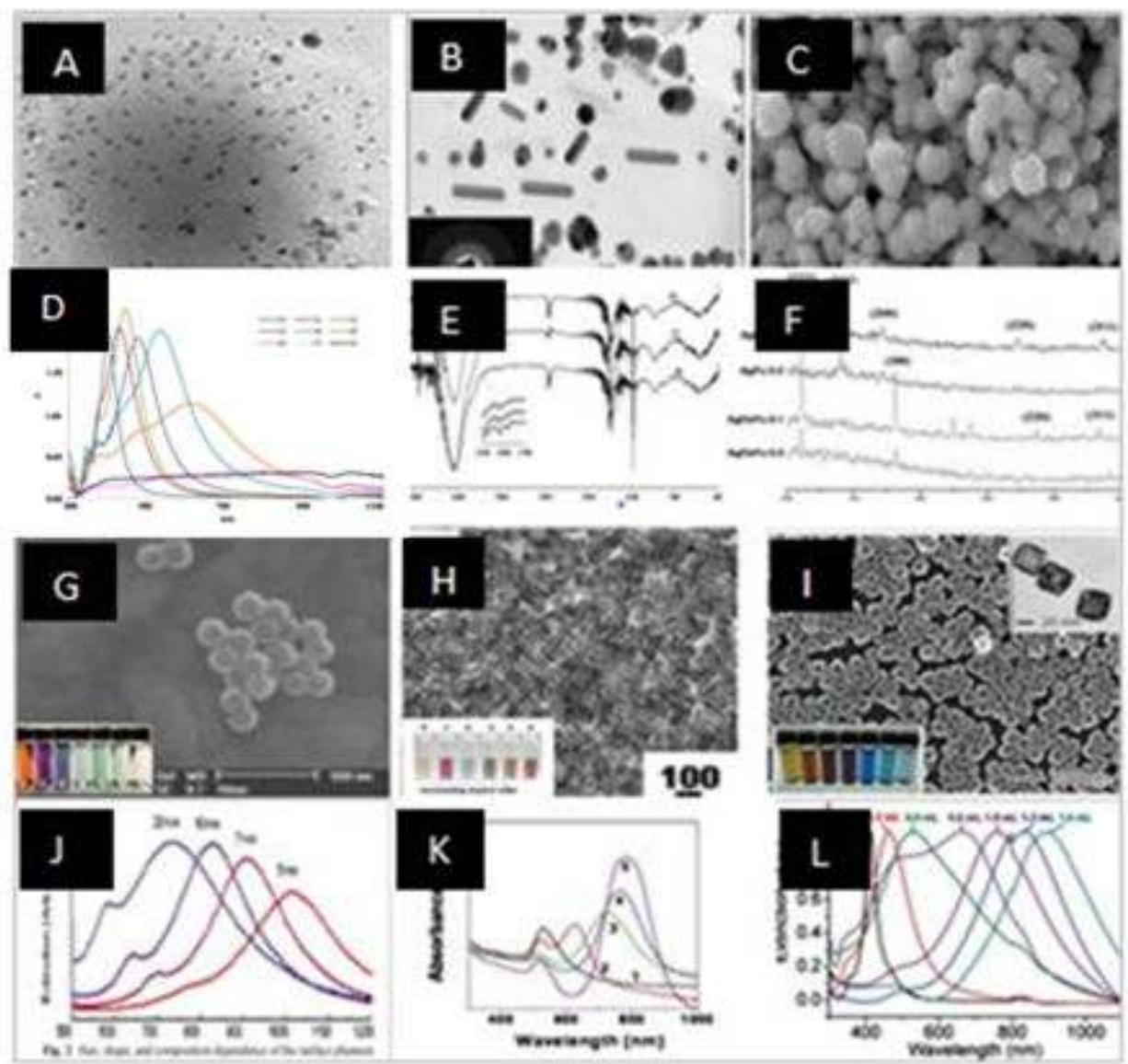

Figure 2. A: TEM image of methyl cellulose-capped silver nanoparticles at $60{ }^{\circ} \mathrm{C}$ [89]; B: TEM image of methyl cellulose-capped silver nanoparticles at $90{ }^{\circ} \mathrm{C}$ [89]; C: SEM image of starch-stabilized silver nanoparticles [90]; D: UV-Vis for starch-capped silver nanoparticles [90]; E: FTIR of starch-capped silver nanoparticles [90]; F: XRD for pullulan/ oxidized pullulan-stabilized silver nanoparticles [82]; Some gold nanostructures (Top): nanoshells (G) [115], nanorods (H) [114] nanocages (I) [91] and their corresponding SPR characteristics (J-L) (Below). 

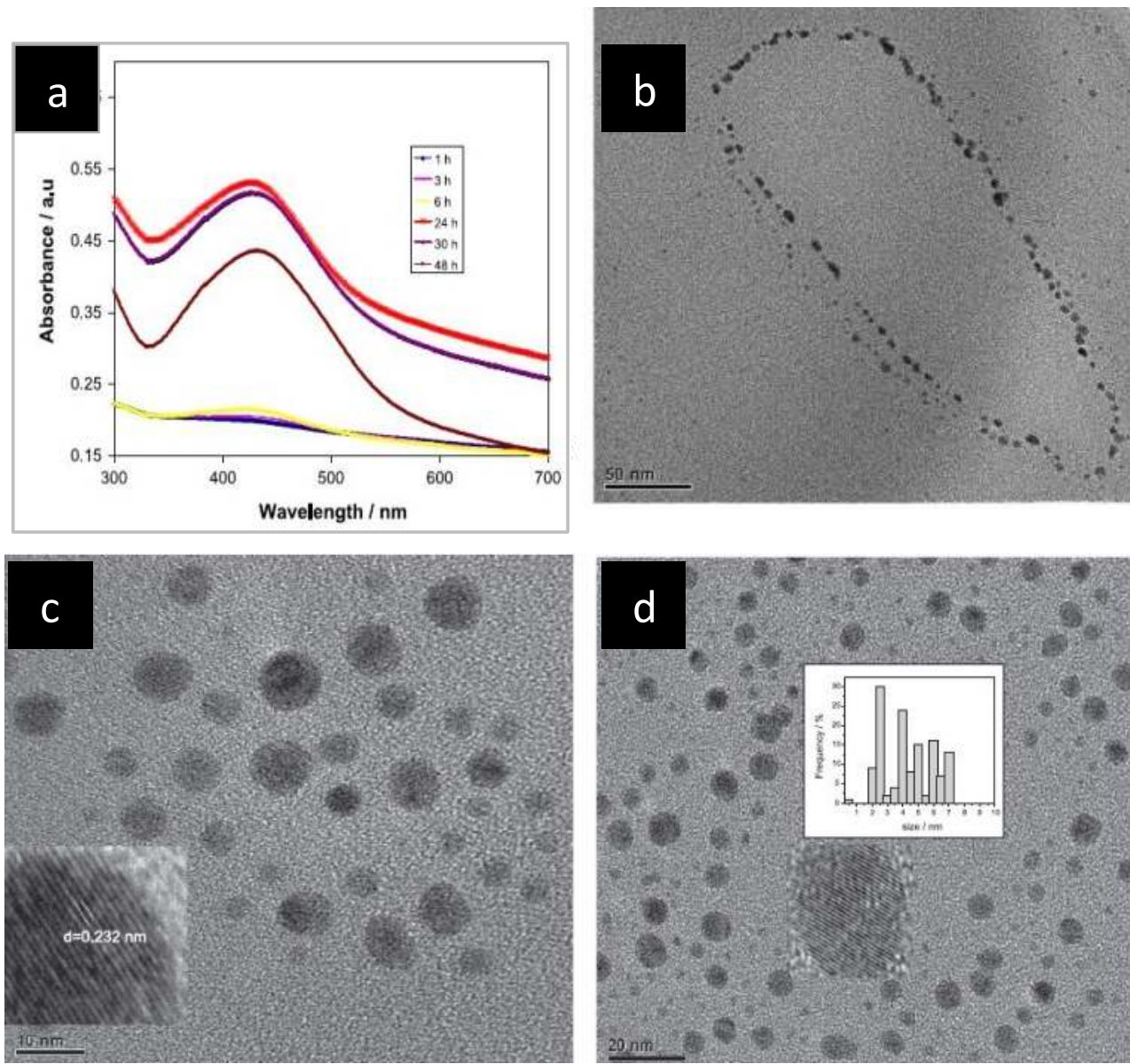

Figure 3. Elucidation of a reaction mechanism using UV-Vis and TEM. a: UV-Vis absorption spectra of Ag-NPs at different reaction times during the synthesis of Ag-NPs. b: TEM image of Ag-NPs (at $1 \mathrm{~h}$ reaction time) showing necklace arrangement. c: TEM image at $24 \mathrm{~h}$ reaction time, scale bar, $10 \mathrm{~nm}$ (inset shows high-resolution image). d: TEM image at $30 \mathrm{~h}$ reaction time, scale bar, $20 \mathrm{~nm}$ (inset shows high-resolution image and particle-size distribution) [81].

\section{Biomedical applications of polysaccharide-stabilized gold and silver nanoparticles}

\subsection{Gold nanoparticles}

Gold nanoparticles are essential nanomaterials for many biomedical applications such as targeted biological sensing [94], site-specific drug delivery, diagnostic imaging $[84,95,96]$ and 
photothermal therapy $[95,97]$. They are essentially utilized for these applications due to their unique SPR property, inertness [84,96], non-toxicity, enhanced permeability retention effect, conjugatable surfaces for the attachment of targeting [98] and therapeutic agents [99], nearinfrared light absorption [97], high light absorption [95] and scattering properties and generation of therapeutic heat after light irradiation [95]. Recent advances in the use of gold nanoparticles have focused on the use of polysaccharide-stabilized gold nanoparticles. One of the reasons for this is the better efficiency of polysaccharides in synthesizing and stabilizing gold nanoparticles compared to other stabilizing agents such as oligosaccharides or monosaccharides [100]. Other reasons are their low toxicity, easily conjugatable functional groups [101] and good biocompatibility [102,103]. Some of the recent biomedical applications of gold nanoparticles are provided in the next section.

\subsubsection{Targeted plasmonic biosensing detection}

Various structures of gold nanoparticles such as the nanospheres, nanorods and nanocages have been shown to exhibit different SPR characteristics [78,103], which can be used to detect biomarkers [104] and pathogens in body fluid samples. In targeted plasmonic-assisted biosensing, stabilized gold nanoparticles are conjugated to some specific biomolecule targeting agents such as carbohydrates [102,105] peptides [106], antibodies and oligonucleotides [102] which react specifically with some disease biomarkers or microbes. This interaction often leads to the aggregation of gold nanoparticles [104] which causes shifting of the surface plasmon resonance peak. This shift effect can be detected by visual colour change, spectrophotometric measurement or dynamic light scattering techniques. Targeted plasmonic biosensing could be successfully employed for the rapid improvement of various diagnostic techniques such as homophase immunoassay, DNA assay and enzymatic assay [104].

\subsubsection{Targeted bioimaging enhancement}

Gold nanoparticles are plasmonic entities which absorb and scatter light from the visible to near-infrared optical region where tissues are relatively transparent to light $[106,107]$. These properties enable them to be used as contrasting agents in many biomedical imaging applications such as magnetic resonance imaging and computed tomography [108]. When excited with near-infrared light, gold nanoparticles absorb and scatter light more than the surrounding tissues leading to a better detection of their sites of localization. In targeted magnetic resonance imaging (MRI) technique, stabilized gold NPs are conjugated to an active targeting material such as a specific site-targeting carbohydrate [105], peptides [106,109,110], aptamers [108] or external magnetic field-driven iron oxide nanoparticles (IONPs) [111] in order to achieve active site-targeting, detection and better imaging. Superparamagnetic iron oxide nanoparticles (SPIONs) are excellent MRI contrast agents [112,113]. Thus, utilizing stabilized gold-SPION hybrids, such as SPION-gold core-shell, promotes greater improvement of the MRI technique. Furthermore, gold NPs may be coupled with conventional imaging dyes or contrasting agents to improve their imaging efficacies. 


\subsubsection{Targeted fluorescence detection}

In targeted fluorescence detection, stabilized gold nanoparticles are either bonded directly [98] or conjugated to a fluorescence molecule [95] bonded to an active site-targeting biomolecule [110]. The technique makes use of (i) the synergetic properties of the selective localization of the gold nanoparticles in disease tissues, (ii) interaction of the active targeting biomolecules with their corresponding disease tissue receptors and (iii) emitted fluorescence light from the gold nanoparticles [98] or conjugated fluorophore to improve detection of disease tissues [110]. For diagnosis purposes, the active site targeting gold nanoparticle solution may be injected via intravenous means and the body exposed to the fluorescence spectroscopy imaging technique. The diseased tissues containing the gold nanoparticle fluorophore conjugates will emit light of specific wavelength while the healthy tissue remains non-fluorescent $[95,110]$.

\subsubsection{Targeted Photothermal Therapy (PTT)}

Targeted PTT involves the use of active site targeting stabilized gold nanoparticles (Au-NPs) for specific tumour cell destructions. The Au-NPs generate therapeutic heat after irradiation with light of appropriate wavelength and dose. The site targeting agent interacts with the tumour cell surfaces in order to pave way for the nanoparticles to gain entrance into the cell where the therapeutic function will take place. Especially suitable for this purpose are the gold nanorods $[78,114]$ and nanoshells $[78,115]$ whose aspect ratio and the ratio of the shell to core diameter can be tuned to absorb light in the near-infrared regions where biological tissues are transparent to light. Gold nanorods have been extensively applied for the eradication of cancer cells via PTT [102]. Other gold nanoparticle structures that can be used for PTT are gold nanocages [78,91]. However, all gold nanostructures exhibit different PTT efficacies due to their different structures and surface plasmon resonance properties.

\subsection{Silver nanoparticles}

\subsubsection{Diagnostic enhancement}

Stabilized silver nanoparticles (Ag-NPs), being a plasmonic entity also exhibit biological imaging characteristic $[96,116]$ and plasmonic and fluorescence detection of biomolecules $[116,117,118]$ and toxic heavy metals $[119,120]$. Like gold nanoparticles, it could be used to improve image contrast of disease tissues when functionalized or employed as nanocarriers of specific site-targeting agents such as peptides, antibodies and carbohydrates which show some specific affinity towards certain molecules present in disease tissues. As nanocarriers of these biomolecules, silver nanoparticles may cause enhanced biological image contrast via their light absorption and scattering properties. However, the choice of silver nanoparticles for fluorescence imaging function may be more for silver nanoclusters (Ag-NCs) compared to other silver morphologies due to their small size-dependent fluorescence effect. It has been reported that as the size of silver nanoparticles approaches the dimension of the Fermi wavelength of an electron, they emit fluorescence light which can be tuned from the visible to near-infrared region after suitable light irradiations [116]. Moreover, Ag-NCs have also been 
found to be useful as an excellent nanobiosensor agent for the detection of biotoxins [118] and toxic inorganic heavy metals [120]. Nonetheless, like gold nanoparticles, the interaction of functionalized, stabilized silver nanoparticles with disease biomarkers such as proteins may cause aggregation and thus a shift in the position of their SPR band which may be used to detect these biomarkers.

\subsubsection{Targeted antimicrobial activity}

Silver nanoparticles are especially known for their antibacterial activities [90,121-125]. However, in recent years, reports about the antiviral effects of silver nanoparticles are beginning to emerge [126-128]. In their study, Gusseme and co-workers [128] employed biogenic silver nanoparticles to remove murine norovirus 1 (MNV-1) from drinking water. The biogenic silver was mixed with a water filter to effect the viral removal. Also, the use of silver nanoparticles in preventing the binding of HIV-1 to human host cells via binding of nanoparticles to the virus' glycoproteins in vitro has been reported [129]. The sulphur group of the virus' gp120 glycoprotein has been suggested to be the binding target site $[129,130]$. Furthermore, the applications of silver nanoparticles such as antifungal [131] and antiplasmodium agents [132] have also been reported.

\subsubsection{Anti-angiogenesis activity}

Silver nanoparticles could also function as anti-angiogenesis agents by preventing the formation of new blood vessels responsible for the development of many human diseases such as cancer, macular degeneration and inflammatory diseases [133]. In their study, Gurunathan and co-workers [133] suggested that silver nanoparticles readily interfere with the angiogenesis pathway by inhibiting the activation of p13k/akt.

\section{Conclusion}

Biopolymers have continued to revolutionize the advancements of green nanotechnology. Nanoscale derivatives of polymers like starch, cellulose and dextran can be synthesized on a large scale and can be used for the manufacture of bionanocomposites. They present promising alternatives to the environmental challenging non-biodegradable plastics via industrially viable process. Most of these biopolymers also offer a route for completely green synthesis of several noble metal nanoparticles performing the dual role of stabilizing and reducing agents in aqueous medium. Biopolymer-mediated synthesis offers numerous advantages which include efficient solubility in water, non-cytotoxicity and biocompatibility in biomedical applications. Polysaccharide-capped silver and gold nanoparticles are emerging as excellent nanomaterials in many fields, including biomedical and environmental. This is due to the result of the excellent functionalities inferred on them by the polysaccharide molecules. These properties include long-term stability and high solubility in aqueous solution, specific biomolecule targeting which induces specific therapeutic functions and toxicity reduction. 


\section{Author details}

Olayemi J. Fakayode ${ }^{1,2}$, Adewale O. Oladipo ${ }^{1,2}$, Oluwatobi S. Oluwafemi ${ }^{1,2^{*}}$ and Sandile P. Songca ${ }^{3}$

*Address all correspondence to: oluwafemi.oluwatobi@gmail.com

1 Department of Applied Chemistry, University of Johannesburg, Doornfontein, Johannesburg, South Africa

2 Centre for Nanomaterials Science Research, University of Johannesburg, Johannesburg, South Africa

3 Department of Chemistry, Walter Sisulu University, Private bag X1, Mthatha, South Africa

\section{References}

[1] Djoković V, Božanic DK, Vodnik VV., Krsmanović RM, Trandafilovic LV., Dimitrijević-Branković S. Structure and optical properties of noble-metal and oxide nanoparticles dispersed in various polysaccharide biopolymers. Phys Chem Interfaces Nanomater. 2011;18(1):8098-98.

[2] Dos Santos DS, Goulet PJG, Pieczonka NPW, Oliveira ON, Aroca RF. Gold nanoparticle embedded, self-sustained chitosan films as substrates for surface-enhanced Raman scattering. Langmuir. 2004;20(23):10273-7.

[3] Porel S, Venkatram N, Rao DN, Radhakrishnan TP. In situ synthesis of metal nanoparticles in polymer matrix and their optical limiting applications. J Nanosci Nanotechnol. 2007;7(6):1887-92.

[4] B. Goudoulas T. Polymers and biopolymers as drug delivery systems in nanomedicine. Recent Pat Nanomed. 2012;2(1):52-61.

[5] Nitta SK, Numata K. Biopolymer-based nanoparticles for drug/gene delivery and tissue engineering. Int J Mol Sci. 2013;14(1):1629-54.

[6] Liu Z, Jiao Y, Wang Y, Zhou C, Zhang Z. Polysaccharides-based nanoparticles as drug delivery systems. Adv Drug Deliv Rev. Elsevier B.V.2008;60(15):1650-62.

[7] Zhang L, Chan JM, Gu FX, Rhee JW, Wang AZ, Radovic-Moreno AF, et al. Self-assembled lipid-polymer hybrid nanoparticles. A robust drug delivery platform. ACS Nano. 2008;2(8):1696-702.

[8] Johnston H, Brown D, Kermanizadeh A, Gubbins E, Stone V. Investigating the relationship between nanomaterial hazard and physicochemical properties: Informing 
the exploitation of nanomaterials within therapeutic and diagnostic applications. J Control Release. Elsevier B.V. 2012;164(3):307-13.

[9] Farokhzad OC, Langer R. Impact of nanotechnology on drug delivery. ACS Nano. 2009;3(1):16-20.

[10] Tebbe M, Kuttner C, Männel M, Fery A, Chanana M. Colloidally stable and surfactant-free protein-coated gold nanorods in biological media. Appl Mater Interfaces. 2015;7:5984-91.

[11] Li Z, Huang P, Zhang X, Lin J, Yang S, Liu B, et al. RGD-conjugated dendrimermodified gold nanorods for in vivo tumor targeting and photothermal therapy. Mol Pharm. 2010;7(1):94-104.

[12] Alkilany AM, Nagaria PK, Hexel CR, Shaw TJ, Murphy CJ, Wyatt MD. Cellular uptake and cytotoxicity of gold nanorods: Molecular origin of cytotoxicity and surface effects. Small. 2009;5:701-8.

[13] Lewinski N, Colvin V, Drezek R. Cytotoxicity of nanoparticles. Small. 2008;4(1):2649.

[14] Raveendran P, Fu J, Wallen SL. Completely "green" synthesis and stabilization of metal nanoparticles. J Am Chem Soc. 2003;125(46):13940-1.

[15] Wurm FR, Weiss CK. Nanoparticles from renewable polymers. Front Chem. 2014;2(July):49.

[16] Rajan M, Raj V. Potential drug delivery applications of chitosan based nanomaterials. Int Rev Chem Eng. 2013;5(2):145-55.

[17] Wu G, Li P, Feng H, Zhang X, Chu PK. Engineering and functionalization of biomaterials via surface modification. J Mater Chem B. 2015;3(10):2024-42.

[18] Cinelli M, Coles SR, Nadagouda MN, Błaszczyński J, Słowiński R, Varma RS, et al. A green chemistry-based classification model for the synthesis of silver nanoparticles. Green Chem. 2015;17(5):2825-39.

[19] Cabrera FC, Mohan H, dos Santos RJ, Agostini DLS, Aroca RF, Rodríguez-Pérez MA, et al. Green synthesis of gold nanoparticles with self-sustained natural rubber membranes. J Nanomater. 2013; 1-10.

[20] Huang X, Neretina S, El-Sayed MA. Gold nanorods: From synthesis and properties to biological and biomedical applications. Adv Mater. 2009; 21(48):4880-910.

[21] Nath D, Banerjee P. Green nanotechnology - a new hope for medical biology. Environ Toxicol Pharmacol. Elsevier B.V. 2013;36(3):997-1014.

[22] Xie J, Zhang Q, Lee JY, Wang DIC. The synthesis of SERS-active gold nanoflower tags for in vivo applications. ACS Nano. 2008;2:2473-80. 
[23] Sahadev A Shankarappa, Manzoor Koyakutty, Shantikumar V Nair. Efficacy versus toxicity - The ying and yang in translating nanomedicines. Nanomater Nanotechnol. 2014;4(23): 1-9.

[24] Tang XZ, Kumar P, Alavi S, Sandeep KP. Recent advances in biopolymers and biopolymer-based nanocomposites for food packaging materials. Crit Rev Food Sci Nutr. 2012;52(5):426-42.

[25] Sankar MU, Aigal S, Maliyekkal SM, Chaudhary A, Anshup, Kumar AA, et al. Biopolymer-reinforced synthetic granular nanocomposites for affordable point-of-use water purification. Proc Natl Acad Sci USA. 2013;110 (21):8459-64.

[26] Csáki A, Thiele M, Jatschka J, Dathe A, Zopf D, Stranik O, et al. Plasmonic nanoparticle synthesis and bioconjugation for bioanalytical sensing. Eng Life Sci. 2015; 15(3): 266-275.

[27] Joy NA., Janiszewski BK, Novak S, Johnson TW, Oh SH, Raghunathan A, et al. Thermal stability of gold nanorods for high-temperature plasmonic sensing. J Phys Chem C. 2013;117(22):11718-24.

[28] Sung HK, Oh SY, Park C, Kim Y. Colorimetric detection of $\mathrm{Co}^{2+}$ ion using silver nanoparticles with spherical, plate, and rod shapes. Langmuir. 2013;29(28):8978-82.

[29] Siemianowicz K, Likus W, Markowski J. Medical aspects of nanomaterial toxicity. In: Soloneski S, Larramendy ML, editors. Nanomaterials - Toxicity and Risk Assessment. InTech; 2015. p.161-75

[30] González-muñoz M, Díez P, González-gonzález M, Dégano RM, Ibarrola N, Orfao A, et al. Evaluation strategies of nanomaterials toxicity. In: Soloneski S, Larramendy ML, editors. Nanomaterials - Toxicity and Risk Assessment. InTech; 2015. p. 23-37.

[31] Clichici S, Filip A. In vivo assessment of nanomaterials toxicity. In: Soloneski S, Larramendy ML, editors. Nanomaterials - Toxicity and Risk Assessment. InTech; 2015. p. 93-121

[32] Campelo JM, Luna D, Luque R, Marinas JM, Romero AA. Sustainable preparation of supported metal nanoparticles and their applications in catalysis. Chem Sus Chem. 2009;2(1):18-45.

[33] Niu W, Zhang L, Xu G. Seed-mediated growth of noble metal nanocrystals: crystal growth and shape control. Nanoscale. 2013;5(8):3172-81.

[34] Burda C, Chen X, Narayanan R, El-Sayed M A. Chemistry and properties of nanocrystals of different shapes. Chem Rev. 2005;1025-102.

[35] Babu VJ, Vempati S, Uyar T, Ramakrishna S. Review of one-dimensional and two-dimensional nanostructured materials for hydrogen generation. Phys Chem Chem Phy. 2015;17(5):2960-86. 
[36] Tiwari JN, Tiwari RN, Kim KS. Zero-dimensional, one-dimensional, two-dimensional and three-dimensional nanostructured materials for advanced electrochemical energy devices. Prog Mater Sci. 2012;57(4):724-803.

[37] Murphy CJ, Gole AM, Hunyadi SE, Orendorff CJ. One-dimensional colloidal gold and silver nanostructures. Inorg Chem. 2006;45(19):7544-54.

[38] Song Y, Yang Y, Medforth CJ, Pereira E, Singh AK, Xu H, et al. Controlled synthesis of 2-D and 3-D dendritic platinum nanostructures. J Am Chem Soc. 2004;126(2):63545.

[39] Zhang Y, Grady NK, Ayala-Orozco C, Halas NJ. Three-dimensional nanostructures as highly efficient generators of second harmonic light. Nano Lett. 2011;11(12):551923.

[40] Kuchibhatla SVNT, Karakoti AS, Bera D, Seal S. One dimensional nanostructured materials. Prog Mater Sci. 2007;52(5):699-913.

[41] Mohl M, Kumar A, Mohana Reddy AL, Kukovecz A, Konya Z, Kiricsi I, et al. Synthesis of catalytic porous metallic nanorods by galvanic exchange reaction. J Phys Chem C. 2010;114:389-93.

[42] Abdelrasoul GN, Cingolani R, Diaspro A, Athanassiou A, Pignatelli F. Photochemical synthesis: Effect of UV irradiation on gold nanorods morphology. J Photochem Photobiol A Chem. 2014;275:7-11.

[43] Zhu Y, Wang X, Guo W, Wang J, Wang C. Sonochemical synthesis of silver nanorods by reduction of silver nitrate in aqueous solution. Ultrason Sonochem. 2010;17(4): 675-9.

[44] Sathishkumar M, Pavagadhi S, Mahadevan A, Balasubramanian R. Biosynthesis of gold nanoparticles and related cytotoxicity evaluation using A549 cells. Ecotoxicol Environ Saf. 2014;114:232-40.

[45] Ayyad O, Muñoz-Rojas D, Oró-Solé J, Gómez-Romero P. From silver nanoparticles to nanostructures through matrix chemistry. J Nanopart Res. 2010;12(1):337-45.

[46] Turkevich J, Stevenson PC, Hillier J. A study of the nucleation and growth processes in the synthesis of colloidal gold. Discuss Faraday Soc. 1951;11(c):55-75.

[47] Zhao L, Jiang D, Cai Y, Ji X, Xie R, Yang W. Tuning the size of gold nanoparticles in the citrate reduction by chloride ions. Nanoscale. 2012;4(16):5071.

[48] Bastús NG, Merkoçi F, Piella J, Puntes V. Synthesis of highly monodisperse citratestabilized silver nanoparticles of up to $200 \mathrm{~nm}$ : Kinetic control and catalytic properties. Chem Mater. 2014;26(9):2836-46.

[49] Mavani K, Shah M. Synthesis of silver nanoparticles by using sodium borohydride as a reducing agent. Int J Eng Res Technol. 2013;2(3):1-5. 
[50] Deraedt C, Salmon L, Gatard S, Ciganda R, Hernandez R, Ruiz J, et al. Sodium borohydride stabilizes very active gold nanoparticle catalysts. Chem Commun. Royal Society of Chemistry. 2014;50(91):14194-6.

[51] El-Sayed MA. Small Is different: shape-, composition-dependent properties of some colloidal semiconductor nanocrystals. Acc. Chem. Res., 2004; 37(5):326-333.

[52] Lee KS, El-Sayed MA. Dependence of the enhanced optical scattering efficiency relative to that of absorption for gold metal nanorods on aspect ratio, size, end-cap shape, and medium refractive index. J Phys Chem B. 2005;109(43):20331-8.

[53] Samal AK, Sreeprasad TS, Pradeep T. Investigation of the role of $\mathrm{NaBH}_{4}$ in the chemical synthesis of gold nanorods. J Nanopart Res. 2009;12(5):1777-86.

[54] Yong KT, Sahoo Y, Swihart MT, Prasad PN. Synthesis and plasmonic properties of silver and gold nanoshells on polystyrene cores of different size and of gold-silver core-shell nanostructures. Colloids Surf A Physicochem Eng Asp. 2006;290:89-105.

[55] Zhang Z, Xu L, Li H, Kong J. Wavelength-tunable luminescent gold nanoparticles generated by cooperation ligand exchange and their potential application in cellular imaging. RSC Adv. 2013;3(1):59-63.

[56] Kumar R, Korideck H, Ngwa W, Berbeco RI, Makrigiorgos GM, Sridhar S. Third generation gold nanoplatform optimized for radiation therapy. Transl Cancer Res. 2013;2(4):228-39.

[57] Hueso JL, Sebastián V, Mayoral Á, Usón L, Arruebo M, Santamaría J. Beyond gold: rediscovering tetrakis-(hydroxymethyl)-phosphonium chloride (THPC) as an effective agent for the synthesis of ultra-small noble metal nanoparticles and Pt-containing nanoalloys. RSC Adv. 2013;3(26):10427.

[58] Atsushi F, Dhepe PL. Sustainable green catalysis by supported metal nanoparticles. Chem Rec. 2009;9(4):224-35.

[59] Sasaki C, Sumimoto K, Asada C, Nakamura Y. Direct hydrolysis of cellulose to glucose using ultra-high temperature and pressure steam explosion. Carbohydr Polym. 2012;89(1):298-301.

[60] Venkatesham M, Ayodhya D, Madhusudhan A, Veera Babu N, Veerabhadram G. A novel green one-step synthesis of silver nanoparticles using chitosan: catalytic activity and antimicrobial studies. Appl Nanosci. 2012;4(1):113-9.

[61] Ahmed HB, Zahran MK. Facile precursor for synthesis of silver nanoparticles using alkali treated maize starch. Int Sch Res Not. 2014:1-12.

[62] Ayala Valencia G, Cristina de Oliveira Vercik L, Ferrari R, Vercik A. Synthesis and characterization of silver nanoparticles using water-soluble starch and its antibacterial activity on Staphylococcus aureus. Starch - Stärke. 2013;65(11-12):931-7. 
[63] Singh M, Sinha I, Mandal RK. Role of $\mathrm{pH}$ in the green synthesis of silver nanoparticles. Mater Lett. 2009;63(3-4):425-7.

[64] Shervani Z, Yamamoto Y. Carbohydrate-directed synthesis of silver and gold nanoparticles: Effect of the structure of carbohydrates and reducing agents on the size and morphology of the composites. Carbohydr Res. 2011;346(5):651-8.

[65] Katti KK, Kattumuri V, Bhaskaran S, Katti KV, Kannan R. Facile and general method for synthesis of sugar coated gold nanoparticles. Int J Green Nanotechnol Biomed. 2012;29(6):997-1003.

[66] Tongsakul D, Wongravee K, Thammacharoen C, Ekgasit S. Enhancement of the reduction efficiency of soluble starch for platinum nanoparticles synthesis. Carbohydr Res. 2012;357:90-7.

[67] Engelbrekt C, SØrensen KH, Lübcke T, Zhang J, Li Q, Pan C, et al. 1.7 nm platinum nanoparticles: Synthesis with glucose starch, characterization and catalysis. Chem Phys Chem. 2010;11(13):2844-53.

[68] Habibi Y, Lucia LA, Rojas OJ. Cellulose nanocrystals: chemistry, self-assembly, and applications. Chem Rev. 2010;110:3479-500.

[69] Moon RJ, Martini A, Nairn J, Simonsen J, Youngblood J. Cellulose nanomaterials review: structure, properties and nanocomposites. Chem Soc Rev. 2011;40(7):3941-94.

[70] Siqueira G, Bras J, Dufresne A. Cellulosic bionanocomposites: A review of preparation, properties and applications. Polym (Basel). 2010;2(4):728-65.

[71] Hebeish AA., El-Rafie MH, Abdel-Mohdy FA, Abdel-Halim ES, Emam HE. Carboxymethyl cellulose for green synthesis and stabilization of silver nanoparticles. Carbohydr Polym. 2010;82(3):933-41.

[72] Mochochoko T, Oluwafemi OS, Jumbam DN, Songca SP. Green synthesis of silver nanoparticles using cellulose extracted from an aquatic weed; water hyacinth. Carbohydr Polym. 2013;98(1):290-4.

[73] Cai J, Kimura S, Wada M, Kuga S. Nanoporous cellulose as metal nanoparticles support. Biomacromolecules. 2009;10(1):87-94.

[74] De Santa Maria LC, Santos ALC, Oliveira PC, Barud HS, Messaddeq Y, Ribeiro SJL. Synthesis and characterization of silver nanoparticles impregnated into bacterial cellulose. Mater Lett. 2009;63(9-10):797-9.

[75] Wang Y, Zhan L, Huang CZ. One-pot preparation of dextran-capped gold nanoparticles at room temperature and colorimetric detection of dihydralazine sulfate in uric samples. Anal Methods. 2010;2(12):1982.

[76] Saldias C, Leiva A, Valderas J, Radić D. Environmentally friendly synthesis of noble metal nanoparticles assisted by biodegradable dextran-graft-lactone copolymers. Polym Adv Technol. 2014;25(4):372-9. 
[77] Bezugly M, Kutsevol N, Rawiso M, Upr C, Cedex S, Bezugla T. Water-soluble branched copolymers dextran-polyacrylamide and their anionic derivates as matrices for metal nanoparticles in situ synthesis. Chemik. 2012;66(8):862-7.

[78] Huang X, El-Sayed, MA. Plasmonic photo-thermal therapy (PPTT). Alexandria J Med. 2011;47(1):1-9.

[79] Hussain ST, Iqbal M, Mazhar M. Size control synthesis of starch capped-gold nanoparticles. 2009;11:1383-91.

[80] Mandal A, Sekar S, Chandrasekara, N. Vibrational spectroscopic investigation on interaction of sago starch capped silver nanoparticles with collagen: a comparative physicochemical study using FT-IR and FT-Raman. RSC Adv. 2015;5:15763-71.

[81] Oluwafemi SO, Vuyelwa N, Scriba M, Songca, SP. Green controlled synthesis of monodispersed, stable and smaller sized starch-capped silver nanoparticles. Mater Lett. 2013;106:332-6.

[82] Coseri S, Spatareanu A, Sacarescu L, Rimbu C, Suteu D, Spirk S, Harabagiu V. Green synthesis of the silver nanoparticles mediated by pullulan. Carbohydr Polym. 2015;116:9-17.

[83] Tagad CK, Rajdeo KS, Kulkarni A, More P, Aiyer RC, Sabharwal, S. Green synthesis of polysaccharide stabilized gold nanoparticles: chemo catalytic and room temperature operable vapor sensing application. RSC Adv., 2014; 4, 24014-24019.

[84] Vijayakumar S, Ganesan, S. In vitro cytotoxicity assay on gold nanoparticles with different stabilizing agents. J Nanomater. 2012:1-9.

[85] Mochochoko T, Oluwafemi OS, Jumbam DN, Songca SP. Green synthesis of silver nanoparticles using cellulose extracted from an aquatic weed; water hyacinth. Carbohydr Polym. 2013;98(1):290-4.

[86] El-Sheikh MA, El-Rafie SM, Abdel-Halim ES, El-Rafie MH. Green synthesis of hydroxyethyl cellulose-stabilized silver nanoparticles. J Polym. 2013; 1-11.

[87] Gupta NR, Prasad BLV, Gopinath CS, Badiger, MV. A nanocomposite of silver and thermo-associating polymer by a green route: a potential soft-hard material for controlled drug release. RSC Adv. 2014;4:10261-8.

[88] Aramwit P, Bang N, Ratanavaraporn J, Ekgasit, S. Green synthesis of silk sericin-capped silver nanoparticles and their potent anti-bacterial activity. Nanoscale Res Lett. 2014;9(1):1-7.

[89] Bhui DK, Pyne S, Sarkar P, Bar H, Sahoo GP, Misra, A. Temperature controlled synthesis of silver nanostructures of variable morphologies in aqueous methyl cellulose matrix. J Mol Liq. 2011;158(3):170-4. 
[90] Cheng F, Betts JW, Kelly SM, Hector, AL. Green synthesis of highly concentrated aqueous colloidal solutions of large starch-stabilised silver nanoplatelets. Mat Sci Eng C. 2015;46:530-7.

[91] Hu M, Chen J, Li ZY, Au L, Hartland GV, Li X, Marquez M, Xia Y. Gold nanostructures: engineering their plasmonic properties for biomedical applications. Chem Soc Rev. 2006;35(11):1084-94.

[92] Fabricius AL, Duester L, Meermann B, Ternes TA. ICP-MS-based characterization of inorganic nanoparticles-sample preparation and off-line fractionation strategies. Anal Bioanal Chem. 2014; 406(2):467-79.

[93] Hien NQ, Phu DV, Duy NN, Quoc LA. Radiation synthesis and characterization of hyaluronan capped gold nanoparticles. Carbohydr Polym. 2012;89(2):537-41.

[94] Khlebtsov N, Bogatyrev V, Dykman L, Khlebtsov B, Staroverov S. Analytical and theranostic applications of gold nanoparticles and multifunctional nanocomposites. Theranostics. 2013;3(3):167-80.

[95] Jang B, Park J, Tung C, Kim I, Choi Y. Gold nanorod-photosensitizer complex for near-infrared fluorescence imaging and photodynamic/photothermal therapy in vivo. ACS nano. 2011; 5(2):1086-94.

[96] Wolfbeis OS. An overview of nanoparticles commonly used in fluorescent bioimaging. Chem. Soc. Rev., 2015: 44, 4743-4768.

[97] Huang X, Jain PK, El-Sayed IH, El-Sayed MA. Plasmonic photothermal therapy (PPTT) using gold nanoparticles. Lasers Med Sci. 2008;23(3):217-28.

[98] He H, Xie C, Ren J. Nonbleaching fluorescence of gold nanoparticles and its applications in cancer cell imaging. Anal Chem. 2008; 80(15):5951-7.

[99] Lee HJ, Liu Y, Zhao J, Zhou M, Bouchard RR, Mitcham T, Wallace M, Stafford RJ, Li C, Gupta S, and Melancon MP. In vitro and in vivo mapping of drug release after laser ablation thermal therapy with doxorubicin-loaded hollow gold nanoshells using fluorescence and photoacoustic imaging. J Control Release. 2013;172(1):1-17.

[100] Kavita KK, Vijaya K, Sharanya B, Kattesh VK, Kannan, R. Facile and general method for synthesis of sugar coated gold nanoparticles. Int J Green Nanotechnol and Biomed. 2012;29(6):997-1003.

[101] Sathiyanarayanan G, Vignesh V, Saibaba G, Vinothkanna A, Dineshkumar K, Viswanathana MB, Selvin J. Synthesis of carbohydrate polymer encrusted gold nanoparticles using bacterial exopolysaccharide: a novel and greener approach. RSC Adv. 2014;4:22817-27.

[102] Locatelli E, Monaco I, Franchini MC. Surface modifications of gold nanorods for applications in nanomedicine. RSC Adv. 2015;5:21681-99. 
[103] Biju V. Chemical modifications and bioconjugate reactions of nanomaterials for sensing, imaging, drug delivery and therapy. Chem Soc Rev. 2014;43(3):737-962.

[104] Valentini P, Pompa PP. Gold nanoparticles for naked-eye DNA detection: smart. RSC Adv. 2013; 3:19181-90.

[105] Adak AK, Lin H, Lin C, Lin H. Biomolecular chemistry studying carbohydrate - protein interactions. Org Biomol Chem. 2014;12:5563-73.

[106] Oyelere AK, Chen PC, Huang X, El-Sayed IH, El-Sayed MA. Peptide-conjugated gold nanorods for nuclear targeting. Bioconjug Chem. 2007;18(5):1490-7.

[107] Zhou F, Xing D, Ou Z, Wu B., Resasco DE, Chen WR. Cancer photothermal therapy in the near-infrared region by using single-walled carbon nanotubes. J Biomed Opt. 2009;14(2):021009.

[108] Kim D, Jeong YY, Jon S. A drug-loaded aptamer gold nanoparticle bioconjugate for combined CT imaging and therapy of prostate cancer. ACS Nano. 2010;4(7):3689-96.

[109] Chen WH, Chen JX, Cheng H, Chen CS, Yang J, Xu XD, Wang Y, Zhuo RX, Zhang $X Z$. A new anti-cancer strategy of damaging mitochondria by pro-apoptotic peptide functionalized gold nanoparticles. Chem Commun (Cambridge, England). 2013;49(57):6403-5.

[110] Meyers JD, Cheng Y, Broome, AM, Agnes RS, Schluchter MD, Margevicius S, Wang $X$, Kenney ME, Burda C, Basilion JP. Peptide-targeted gold nanoparticles for photodynamic therapy of brain cancer. Particle \& Particle Systems Characterization. 2015;32:448-57.

[111] Wagstaff AJ, Brown SD, Holden MR, Craig GE, Plumb JA, Brown RE, Schreiter N, Chrzanowski W, Wheate NJ. Cisplatin drug delivery using gold-coated iron oxide nanoparticles for enhanced tumour targeting with external magnetic fields. Inorganica Chim Acta. 2012;393:328-33.

[112] Thomas R, Park IK, Jeong YY. Magnetic iron oxide nanoparticles for multimodal imaging and therapy of cancer. Int J Mol Sci. 2013;14:15910-30.

[113] Pereira C, Pereira AM, Rocha M, Freire C, Geraldes CFGC. Architectured design of superparamagnetic $\mathrm{Fe}_{3} \mathrm{O}_{4}$ nanoparticles for application as MRI contrast agents: mastering size and magnetism for enhanced relaxivity. J. Mater Chem B. 2015;3(30):626173.

[114] Murphy CJ, Gole AM, Hunyadi SE, Stone JW, Sisco PN. Alkilany A, Kinard BE, Hankins P. Chemical sensing and imaging with metallic nanorods, Chem. Commun., 2008, 544-557.

[115] Loo C, Lin A, Hirsch L, Lee M-H, Barton J, Halas N, West J, Drezek R. Nanoshellenabled photonics-based imaging and therapy of cancer. Technol Cancer Res Treat. 2004;3(1):33-40. 
[116] Yuan Z, Chen Y, Li H, Chang H, Chen Y. Fluorescent silver nanoclusters stabilized by DNA scaffolds. Chem Commun. 2014;50:9800-15.

[117] Gangula A, Podila R, Ramakrishna M, Karanam L, Janardhana C, Rao AM. Catalytic reduction of 4-nitrophenol using biogenic gold and silver nanoparticles derived from breynia rhamnoides. Langmuir. 2011;27:15268-74.

[118] Chen J, Zhang X, Cai S, Wu D, Chen M, Wang S, Zhang J. A fluorescent aptasensor based on DNA-scaffolded silver-nanocluster for ochratoxin A detection. Biosens Bioelectron. 2014;57:226-31.

[119] Farhadi K, Forough M, Molaei R, Hajizadeh S, Rafipour A. Highly selective $\mathrm{Hg}^{2+}$ colorimetric sensor using green synthesized and unmodified silver nanoparticles. Sens Actuator: B-Chem. 2012;161(1):880-5.

[120] Peng J, Ling J, Zhang X, Bai H, Zheng L, Cao Q, Ding Z. Sensitive detection of mercury and copper ions by fluorescent DNA/Ag nanoclusters in guanine-rich DNA hybridization. Spectrochim Acta A Mol Biomol Spectrosc. 2015;137:1250-7.

[121] Sondi I, Salopek-Sondi B. Silver nanoparticles as antimicrobial agent: a case study on E. coli as a model for Gram-negative bacteria. J Colloid Interface Sci. 2004;275:177-82.

[122] Hernández-Sierra JF, Ruiz F, Cruz Pena DC, Martínez-Gutiérrez F, Martínez AE, Guillén ADJP, Tapia-Pérez H, Castañón GM. The antimicrobial sensitivity of Streptococcus mutans to nanoparticles of silver, zinc oxide, and gold. Nanomedicine. 2008;4:237-40.

[123] Kaviyaa S, Santhanalakshmi J, Viswanathanb B, Muthumary J, Srinivasan K. Biosynthesis of silver nanoparticles using citrus sinensis peel extract and its antibacterial activity. Spectrochim Acta. 2011 (Part A); 79: 594-59.

[124] El-Sherif H, El-Masry M, Kansoh A. Hydrogels as template nanoreactors for silver nanoparticles formation and their antimicrobial activities. Macromol Res. 2011;19(11):1157-65.

[125] Velmurugan P, Cho M, Lee SM, Park JH, Bae S, Oh BT. Antimicrobial fabrication of cotton fabric and leather using green-synthesized nanosilver. Carbohydr Polym. 2014;106:319-25.

[126] Speshock JL, Murdock RC, Braydich-Stolle LK, Schrand AM, Hussain SM. Interaction of silver nanoparticles with Tacaribe virus. J Nanobiotechnology. 2010;8(19):1-9.

[127] Gusseme BD, Sintubin L, Baert L, Thibo E, Hennebel T, Vermeulen G, Uyttendaele M, Verstraeten W, Boon N. Biogenic silver for disinfection of water contaminated with viruses. Appl Environ Microbiol. 2010;76(4):1082-7.

[128] Bart De Gusseme BD, Hennebel T, Christiaens E, Saveyn H, Verbeken K, Fitts JP, Boon N, Verstraete W. Virus disinfection in water by biogenic silver immobilized in polyvinylidene fluoride membranes. Water Res. 2011;45: 1856-64. 
[129] Elechiguerra JL, Burt JL, Morones JR, Camacho-Bragado A, Gao X, Lara HH, Yacaman MJ. Interaction of silver nanoparticles with HIV-1. J Nanobiotechnology. 2005;3(6):1-10.

[130] Lara HH, Ayala-Nuñez NV, Ixtepan-Turrent L, Rodriguez-Padilla C. Mode of antiviral action of silver nanoparticles against HIV-1. J Nanobiotechnology. 2010;8:1-10.

[131] Panáček A, Kolář M, Večeřová R, Prucek R, Soukupová J, Kryštof V, Hamal P, Zbořil R, Kvítek L. Antifungal activity of silver nanoparticles against Candida spp. Biomater. 2009;30(31):6333-40.

[132] Ponarulselvam S, Panneerselvam C, Murugan K, Aarthi N, Kalimuthu K, Thangamani S. Synthesis of silver nanoparticles using leaves of Catharanthus roseus Linn. G. Don and their antiplasmodial activities. Asian Pac J Trop Biomed. 2012:574-80.

[133] Gurunathan S, Lee KJ, Kalishwaralal K, Sheikpranbabu S, Vaidyanathan R, Eom SH. Antiangiogenic properties of silver nanoparticles. Biomater. 2009;30:6341-50.

[134] Tsuzuki T. Commercial scale production of inorganic nanoparticles, Int J Nanotechnol. 2009;6(5/6): 567-78. 